\title{
Lower Levels of Urinary Nerve Growth Factor Might Predict Recurrent Urinary Tract Infections in Women
}

\author{
Fei-Chi Chuang ${ }^{1}$, Hsin-Tzu Liu', Hann-Chorng Kuo \\ ${ }^{1}$ Department of Obstetrics and Gynecology, Kaohsiung Chang Gung Memorial Hospital and Chang Gung University College of Medicine, Kaohsiung, Taiwan \\ ${ }^{2}$ Department of Urology, Buddhist Tzu Chi General Hospital and Buddhist Tzu Chi University, Hualien, Taiwan
}

\begin{abstract}
Purpose: To investigate the changes in urinary nerve growth factor (uNGF) levels after acute urinary tract infection (UTI) and to assess the role of UNGF in predicting UTI recurrence in women.

Methods: Women with uncomplicated, symptomatic UTIs were enrolled. Cephalexin $500 \mathrm{mg}$ (every 6 hours) was administered for 7-14 days to treat acute UTIs. Subsequently, the patients were randomized to receive either sulfamethoxazole/trimethoprim $800 \mathrm{mg} / 160 \mathrm{mg}$ daily at bedtime, or celecoxib $200 \mathrm{mg}$ daily for 3 months and were monitored for up to 12 months. NGF levels in the urine were determined at baseline, 1, 4, and 12 weeks after the initiation of prophylactic therapy, and were compared between women with first-time UTIs and recurrent UTIs, sulfamethoxazole/trimethoprim and celecoxib-treated women, and no UTI recurrence and UTI recurrence that occurred during the follow-up period. Twenty women free of UTIs served as controls.

Results: A total of 139 women with UTI and 20 controls were enrolled in the study, which included 50 women with a firsttime UTI and 89 women with recurrent UTIs. Thirty-seven women completed the study. Women with recurrent UTIs $(n=23)$ had a trend of lower uNGF levels than women with first-time UTIs $(n=14)$. During follow-up, 9 women had UTI recurrence. The serial uNGF levels in women with UTI recurrence were significantly lower than those in women who did not have UTI recurrence during the follow-up period.

Conclusions: The lower levels of UNGF in women with recurrent UTI and the incidence of UTI recurrence during follow-up suggest that lower uNGF might reflect the defective innate immunity in women with recurrent UTI.
\end{abstract}

Keywords: Biomarkers; Inflammation; Immunity, Innate; Nerve Growth Factor; Urinary Tract Infection

- Research Ethics: The study was approved by the Institutional Review Board and Ethics Committee of the Buddhist Tzu Chi General Hospital and was registered at ClinicalTrials.gov (ClinicalTrials.gov Identifier: NCT01800799).

- Conflict of Interest: No potential conflict of interest relevant to this article was reported.

\section{INTRODUCTION}

Urinary tract infections (UTIs) are common bacterial infections in women, and UTI recurrence occurs in one in four affected women [1]. Recurrent UTI is one of the most common diagnoses of pelvic floor dysfunction among women. Accord- ing to the International Urogynecological Association/ International Continence Society joint report on the terminology for female pelvic floor dysfunction, recurrent UTI is defined as at least 3 symptomatic and medically diagnosed UTIs in a 12month period [2]. The previous UTI should have resolved completely prior to another UTI being diagnosed.

Corresponding author: Hann-Chorng Kuo (iD http://orcid.org/0000-0001-7165-4771 Department of Urology, Buddhist Tzu Chi General Hospital, No. 707, Section 3, Chuang Yang Road, Hualien City, Taiwan

E-mail: hck@tzuchi.com.tw / Tel: +886-3-8561825 / Fax: +886-3-8560794

Submitted: September 26, 2015 / Accepted after revision: December 4, 2015 
Nerve growth factor (NGF) is a small, secreted protein that induces the differentiation and survival of specific target neurons. NGF has a potential role as a urinary biomarker of lower urinary tract dysfunction that has both, diagnostic and therapeutic implications [3-5]. NGF is produced by the urothelium and smooth muscle [6]. Clinical and experimental data directly link increased levels of NGF in the bladder tissue and urine to lower urinary tract disorders, including bladder outlet obstruction, overactive bladder, interstitial cystitis, and chronic prostatitis [7-9]. Increased levels of NGF have also been reported in the bladder tissue and urine of patients with sensory urgency and detrusor overactivity [10-13]. NGF is implicated as a chemical mediator of pathology-induced changes in C-fiber afferent nerve excitability and reflex bladder activity $[14,15]$.

Previous studies have reported that patients with recurrent UTIs have elevated urinary NGF (uNGF), which suggests that chronic inflammation is present in the bladders of these patients after resolution of their UTIs [3]. Chronic inflammation of the bladder wall is associated with increased apoptosis and decreased expression of tight junction proteins of the urothelium [16]. Based on this evidence, we hypothesized that chronic inflammation exists in in the bladder wall after an acute UTI episode, which might potentiate UTI recurrence in these patients. To test this hypothesis, the changes in uNGF levels after acute UTI episodes were assessed. Additionally, we evaluated whether uNGF levels could be used as a biomarker in women to monitor chronic inflammation and predict UTI recurrence following the initial UTI episode.

\section{MATERIALS AND METHODS}

From February 2013 through March 2014, women with uncomplicated, symptomatic, and urine analysis-proven UTI who consecutively visited the outpatient urologic clinic were prospectively enrolled in the study. Patients with previous bladder or urethral surgery, genital prolapse, and possible neurogenic lesions were excluded from the study cohort. The study was approved by the Institutional Review Board and Ethics Committee of the Buddhist Tzu Chi General Hospital and was registered at ClinicalTrials.gov (ClinicalTrials.gov Identifier: NCT 01800799). All patients were informed about the study rationale and procedures, and written consent was obtained before enrollment in the study.

The enrolled women also completed the Chinese version of the self-reported overactive bladder symptom score and visual analogue scale for pain at baseline. Cephalexin $500 \mathrm{mg}$ every 6 hours was administered for 1 to 2 weeks to treat the acute UTIs. When the acute UTI symptoms subsided and a urine analysis was negative for infection, the women were randomized to receive either sulfamethoxazole/trimethoprim $800 \mathrm{mg} / 160 \mathrm{mg}$ daily at bedtime, or celecoxib $200 \mathrm{mg}$ once daily. The women administered antibiotics were treated prophylactically for 3 months while the remaining women were treated for chronic inflammation for 3 months. Uroflowmetry and postvoid residual (PVR) volume were measured before starting the prophylactic antibiotic or anti-inflammatory treatments.

In addition, a $30-\mathrm{mL}$ urine sample at full bladder capacity was obtained for the laboratory testing of uNGF levels at baseline (acute UTI), 1 week (after cephalexin treatment), 4 weeks, and 12 weeks (during and at the end of the prophylactic antibiotic or anti-inflammatory treatments). NGF levels in the urine were measured using an enzyme-linked immunosorbent assay. The changes in UNGF levels were compared between women with first-time UTIs and women with a history of recurrent UTIs, women treated with prophylactic antibiotic and women treated for inflammation after their acute UTI, and women who did not have a UTI recurrence and those that did during the follow-up period.

Recurrent UTI was defined as at least 3 symptomatic and medically diagnosed UTIs in the previous 12 months at the time of patient enrollment. During the follow-up period, UTI recurrence was diagnosed if patient had one episode of UTI proven by urinalysis and urine culture. We also invited 20 women who had stress urinary incontinence and were free of UTIs to serve as controls. All calculations were performed using SPSS ver. 10.0 (SPSS Inc., Chicago, IL, USA). $\mathrm{P}<0.05$ was considered statistically significant.

\section{uNGF Measurements}

The women provided urine samples $(30 \mathrm{~mL})$ at full bladder capacity for laboratory testing of uNGF levels. The UNGF levels were measured using an enzyme-linked immunosorbent assay on undiluted urine samples as previously reported [14]. The uNGF concentration was determined using the Emax ImmunoAssay System (Promega, Madison, WI, USA) with a minimum sensitivity of $7.8 \mathrm{pg} / \mathrm{mL}$ of NGF. The amount of NGF in each urine sample was determined from a standard curve. All samples were tested in triplicate, and the values were averaged. 


\section{RESULTS}

A total of 139 women with UTI and 20 controls were enrolled in the study. Of these, 50 women had a first-time UTI and 89 women had recurrent UTIs. Table 1 shows the baseline demo- graphics and clinical characteristics among the control, firsttime UTI, and recurrent UTI groups. The mean age was similar between the 3 groups (range, 49.4-58.7 years). At the baseline (acute UTI episode), the UNGF levels, overactive bladder symptom score, visual analogue score for pain, maximum urinary

Table 1. Baseline uNGF levels among control, first-time UTI and recurrent UTI groups

\begin{tabular}{|c|c|c|c|c|}
\hline Variable & Control $(\mathrm{n}=20)$ & First-time UTI $(\mathrm{n}=50)$ & Recurrent UTI $(\mathrm{n}=89)$ & P-value \\
\hline Age $(y r)$, mean \pm SD (range) & $49.4 \pm 6.5(41-63)$ & $56.9 \pm 18.7(20-94)$ & $58.7 \pm 17.3(20-89)$ & 0.556 \\
\hline $\mathrm{uNGF}(\mathrm{pg} / \mathrm{mL})$ & $1.59 \pm 4.91$ & $49.68 \pm 84.22^{\mathrm{a})}$ & $37.59 \pm 70.35^{\mathrm{a})}$ & 0.438 \\
\hline OABSS & $2.24 \pm 1.09$ & $4.76 \pm 2.96^{\mathrm{a})}$ & $5.16 \pm 2.58^{\mathrm{a})}$ & 0.405 \\
\hline VAS & $0.03 \pm 0.24$ & $1.55 \pm 2.57^{\mathrm{a})}$ & $1.74 \pm 2.46^{\mathrm{a})}$ & 0.680 \\
\hline Qmax at 1 week $^{\text {b) }}(\mathrm{mL} / \mathrm{sec})$ & - & $20.54 \pm 10.80$ & $20.99 \pm 11.36$ & 0.847 \\
\hline VV at 1 week $^{\mathrm{b})}(\mathrm{mL})$ & - & $290.7 \pm 186.9$ & $257.3 \pm 159.7$ & 0.336 \\
\hline PVR at 1 week $^{\mathrm{b})}(\mathrm{mL})$ & - & $33.36 \pm 56.30$ & $37.39 \pm 63.52$ & 0.747 \\
\hline OABSS at 1 week $^{\mathrm{b})}$ & - & $3.36 \pm 2.06$ & $4.01 \pm 2.35$ & 0.144 \\
\hline
\end{tabular}

Values are presented as mean \pm standard deviation $(\mathrm{SD})$ unless otherwise indicated. P-values indicate the difference between first-time and recurrent UTI groups.

uNGF, urinary nerve growth factor; UTI, urinary tract infection; OABSS, overactive bladder symptom score; VAS, visual analogue score; Qmax, maximum flow rate; VV, voided volume; PVR, postvoid residual volume.

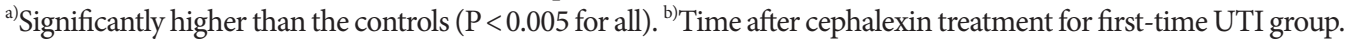

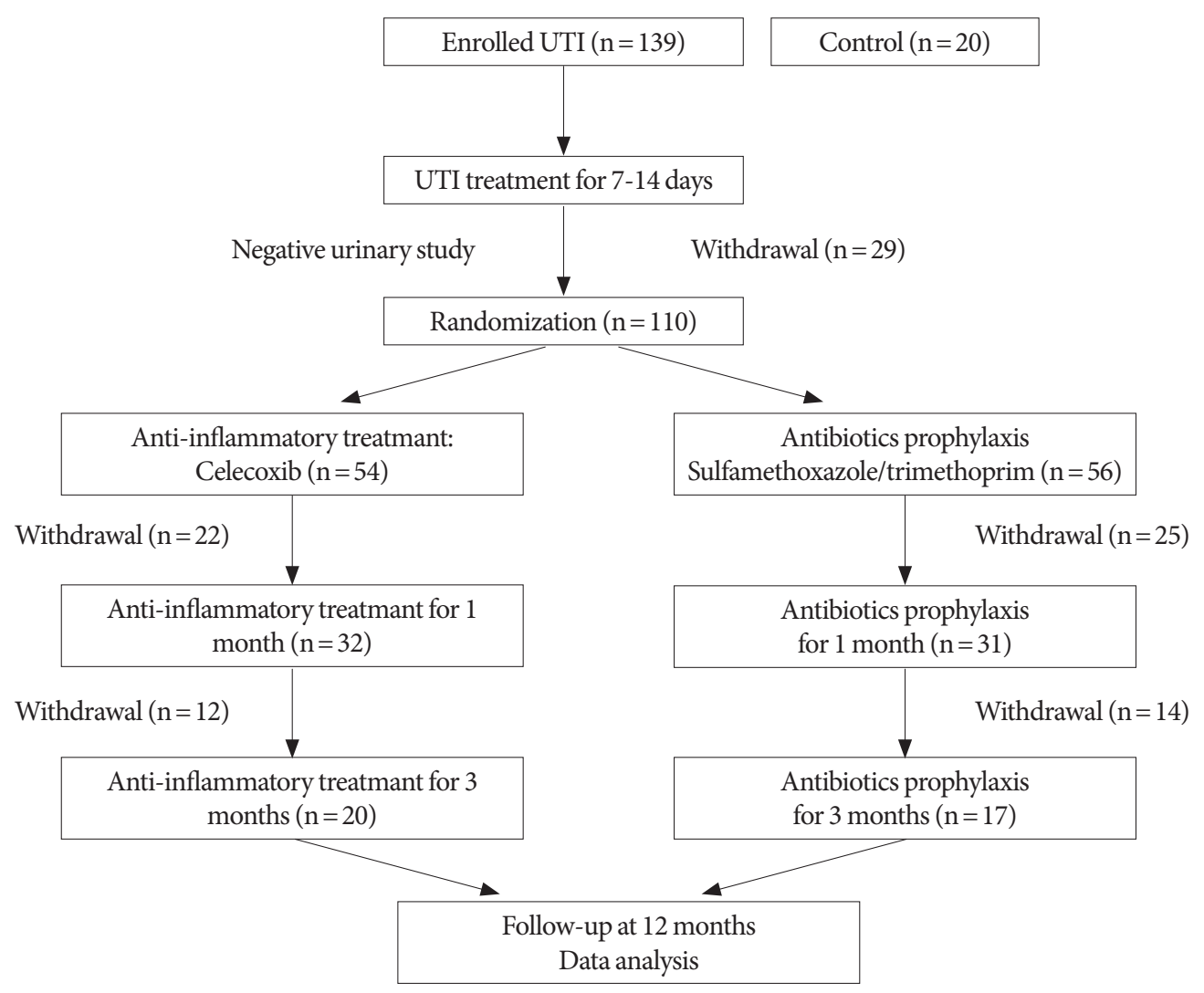

Fig. 1. Flow chart for patient recruitment, randomization, withdrawals and follow-up. 
flow rate, voided urine volume, and PVR volume did not differ significantly between the first-time UTI and recurrent UTI groups.

At the end of the study, only 37 of the 139 women initially enrolled completed the protocol. The information on patient flow is detailed in Fig. 1. The mean uNGF levels of all women with UTIs decreased from $52.6 \pm 77.5 \mathrm{pg} / \mathrm{mL}$ at baseline to $22.4 \pm 38.1 \mathrm{pg} / \mathrm{mL}$ at 4 weeks to $14.4 \pm 24.8 \mathrm{pg} / \mathrm{mL}$ at 12 weeks

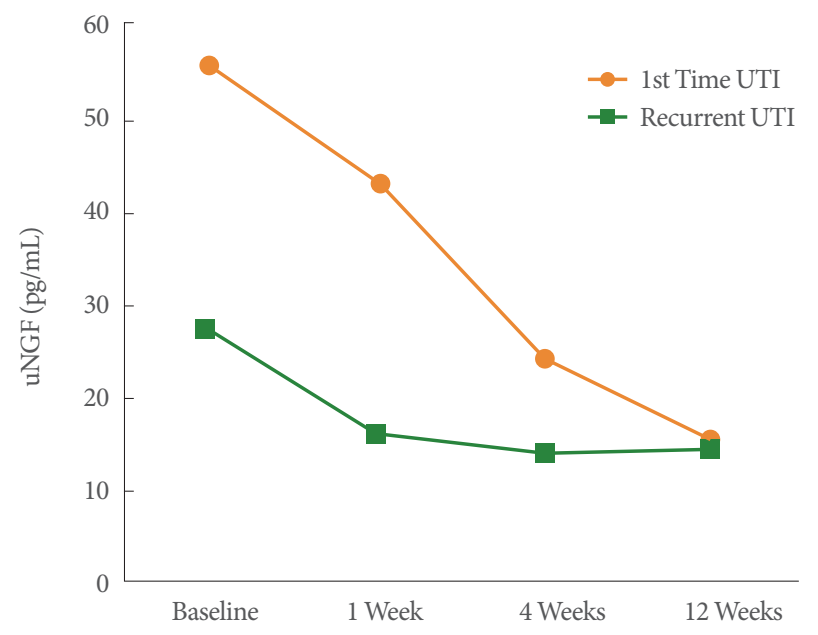

Fig. 2. Serial urinary nerve growth factor (uNGF) levels at baseline, week 1,4 , and 12 in the first-time UTI $(n=14)$ and recurrent UTI $(n=23)$ groups. UTI, urinary tract infection. (all $\mathrm{P}<0.05)$. The mean baseline uNGF level among the women with acute UTIs was significantly higher than that of the controls $(52.6 \pm 77.5 \mathrm{pg} / \mathrm{mL}$ vs. $1.59 \pm 4.91 \mathrm{pg} / \mathrm{mL})$. Although the uNGF levels in women with UTIs declined with time, the levels remained significantly higher after 3 months of antibiotic prophylaxis or anti-inflammatory treatment than those in the controls (Table 1). A comparison between the uNGF levels in the groups with first-time UTIs $(\mathrm{n}=14)$ and recurrent UTIs $(\mathrm{n}=$ 23) showed no significant differences at baseline or weeks 1,4 , and 12 (Table 2). However, we observed a trend of lower uNGF levels in women with recurrent UTIs than in women with a first-time UTI (Fig. 2).

The uNGF levels at baseline, week 1, week 4, and week 12 after cephalexin treatment for the acute UTI did not differ between the groups administered sulfamethoxazole/trimethoprim prophylaxis $(n=17)$ and celecoxib prophylaxis $(n=20)$. Neither drug had any effect in reducing uNGF levels. The serial changes in uNGF levels were also similar between the 2 groups treated with different medications for UTI prophylaxis (Table 3).

During the follow-up period of up to 1 year, 9 women had UTI recurrence. Seven women in the recurrent UTI group at baseline experienced another recurrent UTI (7 of 23, 30.4\%) during the follow-up period. The rate of UTI recurrence was numerically lower among the women in the initial first-time UTI group (2 of 14, 14.3\%). Table 4 presents the serial compari-

Table 2. Serial comparison of uNGF levels between the first-time UTI and recurrent UTI groups

\begin{tabular}{lcccc}
\hline Parameter $(\mathrm{pg} / \mathrm{mL})$ & Controls $(\mathrm{n}=20)$ & First-time UTI $(\mathrm{n}=14)$ & Recurrent UTI $(\mathrm{n}=23)$ & P-value \\
\hline uNGF-baseline & $1.59 \pm 4.91$ & $55.47 \pm 58.36$ & $27.27 \pm 39.56$ & 0.075 \\
uNGF-1 week & - & $42.88 \pm 106.26$ & $15.88 \pm 27.04$ & 0.367 \\
uNGF-4 weeks & - & $23.96 \pm 48.80$ & $13.86 \pm 27.38$ & 0.431 \\
uNGF-12 weeks & - & $15.13 \pm 24.76$ & $14.17 \pm 29.33$ & 0.919 \\
\hline
\end{tabular}

Values are presented as mean \pm standard deviation. P-values indicate the difference between first-time and recurrent UTI groups. uNGF-baseline, -1 week, -4 weeks, -12 weeks: time following case enrollment.

uNGF, urinary nerve growth factor; UTI, urinary tract infection.

Table 3. Serial comparison of uNGF levels between sulfamethoxazole/trimethoprim and Celecoxib treated women

\begin{tabular}{lccc}
\hline Parameter $(\mathrm{pg} / \mathrm{mL})$ & Sulfamethoxazole/trimethoprim $(\mathrm{n}=17)$ & Celecoxib $(\mathrm{n}=20)$ & P-value \\
\hline uNGF-baseline & $44.19 \pm 56.43$ & $33.48 \pm 43.03$ & 0.614 \\
uNGF-1 week & $41.96 \pm 100.95$ & $13.91 \pm 21.20$ & 0.291 \\
uNGF-4 weeks & $12.04 \pm 29.71$ & $22.93 \pm 42.42$ & 0.384 \\
uNGF-12 weeks & $16.19 \pm 31.66$ & $13.13 \pm 23.82$ & 0.739 \\
\hline
\end{tabular}

Values are presented as mean \pm standard deviation. uNGF-baseline, -1 week, -4 weeks, -12 weeks: time following case enrollment. uNGF, urinary nerve growth factor. 
Table 4. Serial comparison of uNGF levels between no UTI recurrence and UTI recurrence women during the follow-up period

\begin{tabular}{lccc}
\hline Parameter $(\mathrm{pg} / \mathrm{mL})$ & No UTI recurrence $(\mathrm{n}=28)$ & UTI recurrence $(\mathrm{n}=9)$ & P-value \\
\hline uNGF-baseline & $43.43 \pm 53.09$ & $22.68 \pm 31.12$ & 0.308 \\
uNGF-1 week & $28.69 \pm 78.04$ & $19.46 \pm 34.37$ & 0.735 \\
uNGF-4 weeks & $21.62 \pm 41.85$ & $6.27 \pm 7.45$ & 0.078 \\
uNGF-12 weeks & $18 \pm 31.66$ & $2.98 \pm 7.87$ & 0.021 \\
\hline
\end{tabular}

Values are presented as mean \pm standard deviation. uNGF-baseline, -1 week, -4 weeks, -12 weeks: time following case enrollment. uNGF, urinary nerve growth factor; UTI, urinary tract infection.

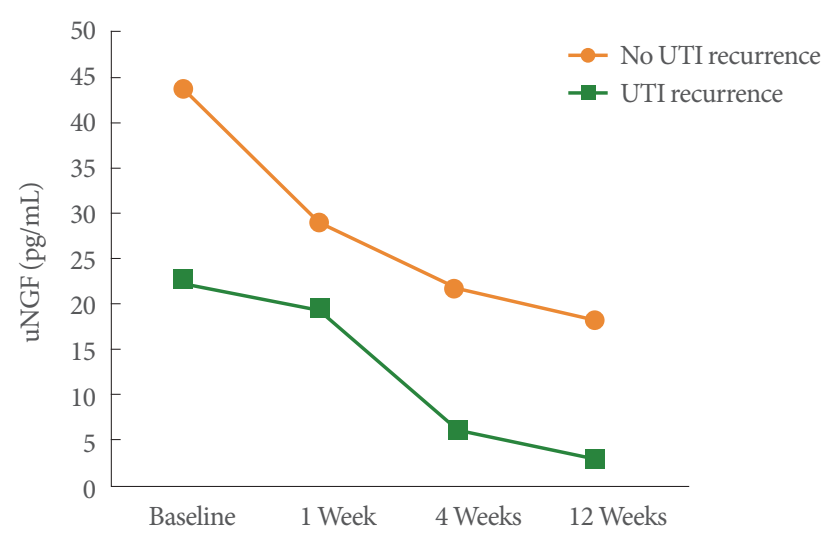

Fig. 3. Serial urinary nerve growth factor (uNGF) levels at baseline, week 1,4 , and 12 in the groups with UTI recurrence $(\mathrm{n}=9)$ and without UTI recurrence $(\mathrm{n}=28)$ during the follow-up period. UTI, urinary tract infection.

son of the UNGF levels between the women that had no UTI recurrence $(n=28)$ and those that had UTI recurrence $(n=9)$ during follow-up. The serial uNGF levels in women with UTI recurrence were lower than that in women without UTI recurrence (Fig. 3). At 12 weeks, the uNGF levels in the group without UTI recurrence were $18 \pm 31.66 \mathrm{pg} / \mathrm{mL}$ vs. $2.98 \pm 7.87 \mathrm{pg} /$ $\mathrm{mL}(\mathrm{P}=0.021)$ in the group with UTI recurrence.

\section{DISCUSSION}

This study demonstrates that uNGF levels significantly increase after an acute episode of UTI and decline with time, but remain higher than the control levels at 3 months after treatment of the acute UTI. The most important finding of this study is that in addition to serving as a biomarker of inflammation, the uNGF level appears to reflect an innate immune response to infection in the women with recurrent UTIs.

During an acute episode of UTI, inflammation of the bladder mucosa and underlying nerves results in uncomfortable ir- ritative symptoms $[17,18]$. It is conceivable that in cases of recurrent UTI, repeated inflammation of the bladder mucosa gradually results in a more chronic pattern of hypersensitivity symptoms even in the absence of bacteriuria. In a previous study, we found that a UTI resulted in increased NGF in the urine, and patients with a resolved UTI but a persistent overactive bladder also had high uNGF levels [3]. We speculate that uNGF is a secondary product of urothelial damage or bladder inflammation that initiates sensory nerve excitation and causes bladder hypersensitivity.

In the present study, we observed that uNGF levels did not decrease to the level of controls even after 3 months of antibiotic or anti-inflammatory treatment. Although uNGF could be reduced in patients with resolved UTIs and no irritative symptoms, the inflammation and changes of urothelial homeostasis might still be present. Additionally, long-term anti-inflammatory therapy may be necessary to prevent a recurrent UTI. Limited information on urothelial homeostasis in patients with recurrent UTI is available in the literature. A recent study demonstrated that inflammatory protein expression and urothelial cell apoptosis were remarkably higher, and the barrier function of the urothelium was impaired in patients with recurrent UTIs [19]. It is possible that chronic inflammation in the bladder wall, which causes urothelial dysfunction and defective barrier function, could potentiate UTI recurrence.

In the present study, uNGF levels were significantly elevated in acute UTIs. After resolution of the acute UTI by cephalexin $500 \mathrm{mg}$ every 6 hours for 1 to 2 weeks, the women were randomized to receive sulfamethoxazole/trimethoprim or celecoxib for 3 months for UTI prophylaxis or lowering inflammation, respectively $[20,21]$. Serial follow-up of uNGF levels showed that though the high uNGF levels associated with the acute UTI gradually declined, they continued to remain above the level in controls even 12 weeks after resolution of the initial UTI episode. This observation shows that even with 3 months of con- 
tinuous antimicrobial prophylaxis or nonsteroid anti-inflammatory therapy, the bladder inflammatory reaction was not completely eradicated.

Recurrent UTIs are vexing problems. Previously, investigators have reported that $25 \%$ of women experience a UTI recurrence within 6 months after their initial UTI episode [22-24]. The present study found that the rate of UTI recurrence was higher in women with recurrent UTIs (7 of 23, 30.4\%) than in the women with first-time UTIs (2 of 14, 14.3\%) during the follow-up period. Indeed, some women appear to be more vulnerable to UTI recurrence.

In the present study, we compared the serial levels of uNGF between the first-time UTI and recurrent UTI groups at baseline, as well as between the groups with and without UTI recurrence during the follow-up period. We found lower levels of uNGF in the groups with recurrent UTI during the follow-up period, which is not consistent with our hypothesis that UNGF reflected more severe residual inflammation in recurrent UTI. The lower levels of UNGF in women with recurrent UTI at baseline and in women with UTI recurrence during the followup period might imply that uNGF may reflect the involvement of innate immunity in recurrent UTIs. Increased uNGF levels might result from increased local immune function and excitation of sensory innervation during a UTI episode. Patients who do not have increased uNGF levels might be more vulnerable to recurrent UTI as a result of lower immune function.

UTI is considered to arise due to complex interactions between the pathogen and the host [25]. Most studies have investigated uropathogenic Escherichia coli (UPEC) because it is by far the most common causative pathogen in UTIs and is detected in $70 \%-95 \%$ of cases $[22,26,27]$. The ability of UPEC to gain a foothold in the bladder is greatly facilitated by type 1 pilusmediated attachment to and invasion of bladder epithelial cells [28]. The actions of attachment and invasion activate a cascade of innate host defenses leading to the death and exfoliation of bladder cells and the production of inflammatory mediators [29]. UPEC avoids clearance by invading and replicating within the epithelial cells. Thus, these bacteria serve as a reservoir for recurrent infections. The persistence of UPEC in bladder tissue may subsequently cause recurrent acute infections based on a complex series of dynamic host-pathogen interactions $[28,29]$.

Acute bacterial cystitis damages the urothelium, causing tissue edema and accumulation of large numbers of inflammatory cells in the urothelium and suburothelium. Therefore, these inflammatory cells could start a cascade of tissue inflammation involving several sensory proteins and cytokines, accompanied by pain and hypersensitivity to bladder distension. Although NGF is linked to acute and chronic inflammation in the bladder wall after acute UTI, it is possible that a higher NGF level after a UTI indicates more tissue inflammation and sensory nerve innervation in response to bacterial invasion. These inflammatory reactions, on the other hand, might also imply a higher innate immunity to residual bacteria. Therefore, a lower uNGF level might imply that the host bladder has a lower immunity to recurrent bacterial infection.

The main limitation of this study is that fewer than $30 \%$ of patients completed the study. It was difficult to persuade women with one episode of a UTI to continue medication for 3 months, especially after the acute symptoms subsided. We disclosed the grounds for recurrent UTI on the informed consent form to clarify the study hypothesis to our study subjects. The enrolled subjects also had the rights to withdraw from the study at any time. This is why we could persuade some women with the first-time UTI to join the study, and is also the reason for low complete rate. However, our finding that women with recurrent UTIs have lower levels of uNGF could have useful clinical implications. Lower innate immunity in women recurrent UTIs and lower uNGF levels could predict recurrent UTIs.

In conclusion, the results of the present study revealed that uNGF levels were significantly elevated in the acute stage of UTI. Of additional importance is the observation that uNGF levels were lower in women with recurrent UTI and women with UTI recurrence during the follow-up period. NGF levels in the urine appear predict the status of the innate immune response in recurrent UTI. This evidence provides a new direction for future research in the area of recurrent UTIs.

\section{ACKNOWLEDGEMENTS}

Ms. Huei-Ling Tseng for data collection and statistical analysis.

\section{REFERENCES}

1. Franco AV. Recurrent urinary tract infections. Best Pract Res Clin Obstet Gynaecol 2005;19:861-73.

2. Haylen BT, de Ridder D, Freeman RM, Swift SE, Berghmans B, Lee J, et al. An International Urogynecological Association (IUGA)/International Continence Society (ICS) joint report on the terminology for female pelvic floor dysfunction. Neurourol Urodyn 2010;29:420. 
3. Liu HT, Chen CY, Kuo HC. Urinary nerve growth factor levels in overactive bladder syndrome and lower urinary tract disorders. J Formos Med Assoc 2010;109:862-78.

4. Chuang FC, Liu HT, Wang LY, Kuo HC. Overactive bladder changes with time: a 5-year longitudinal followup of changes in overactive bladder symptoms, urodynamic studies and urinary nerve growth factor levels. J Urol 2014;192:458-63.

5. Liu HT, Chen CY, Kuo HC. Urinary nerve growth factor in women with overactive bladder syndrome. BJU Int 2011;107:799-803.

6. Steers WD, Tuttle JB. Mechanisms of disease: the role of nerve growth factor in the pathophysiology of bladder disorders. Nat Clin Pract Urol 2006;3:101-10.

7. Tuttle JB, Steers WD, Albo M, Nataluk E. Neural input regulates tissue NGF and growth of the adult rat urinary bladder. J Auton Nerv Syst 1994;49:147-58.

8. Lowe EM, Anand P, Terenghi G, Williams-Chestnut RE, Sinicropi DV, Osborne JL. Increased nerve growth factor levels in the urinary bladder of women with idiopathic sensory urgency and interstitial cystitis. Br J Urol 1997;79:572-7.

9. Liu HT, Kuo HC. Urinary nerve growth factor levels are increased in patients with bladder outlet obstruction with overactive bladder symptoms and reduced after successful medical treatment. Urology 2008;72:104-8.

10. Kim JC, Park EY, Seo SI, Park YH, Hwang TK. Nerve growth factor and prostaglandins in the urine of female patients with overactive bladder. J Urol 2006;175:1773-6.

11. Liu HT, Kuo HC. Urinary nerve growth factor level could be a potential biomarker for diagnosis of overactive bladder. J Urol 2008;179: $2270-4$

12. Liu HT, Chancellor MB, Kuo HC. Urinary nerve growth factor levels are elevated in patients with detrusor overactivity and decreased in responders to detrusor botulinum toxin-A injection. Eur Urol 2009;56:700-6.

13. Liu HT, Kuo HC. Urinary nerve growth factor levels are elevated in patients with overactive bladder and do not significantly increase with bladder distention. Neurourol Urodyn 2009;28:78-81.

14. Vizzard MA. Changes in urinary bladder neurotrophic factor mRNA and NGF protein following urinary bladder dysfunction. Exp Neurol 2000;161:273-84.

15. Yoshimura N. Bladder afferent pathway and spinal cord injury: possible mechanisms inducing hyperreflexia of the urinary bladder. Prog Neurobiol 1999;57:583-606.

16. Shie JH, Kuo HC. Higher levels of cell apoptosis and abnormal Ecadherin expression in the urothelium are associated with inflammation in patients with interstitial cystitis/painful bladder syndrome. BJU Int 2011;108(2 Pt 2):E136-41.

17. Cervero F. Sensory innervation of the viscera: peripheral basis of visceral pain. Physiol Rev 1994;74:95-138.

18. Arya LA, Northington GM, Asfaw T, Harvie H, Malykhina A. Evidence of bladder oversensitivity in the absence of an infection in premenopausal women with a history of recurrent urinary tract infections. BJU Int 2012;110:247-51.

19. Chuang FC, Kuo HC. Increased urothelial cell apoptosis and chronic inflammation are associated with recurrent urinary tract infection in women. PLoS One 2013;8:e63760.

20. Lichtenberger P, Hooton TM. Antimicrobial prophylaxis in women with recurrent urinary tract infections. Int J Antimicrob Agents 2011; 38 Suppl:36-41.

21. Keller WR, Kum LM, Wehring HJ, Koola MM, Buchanan RW, Kelly DL. A review of anti-inflammatory agents for symptoms of schizophrenia. J Psychopharmacol 2013;27:337-42.

22. Hooton TM, Stamm WE. Diagnosis and treatment of uncomplicated urinary tract infection. Infect Dis Clin North Am 1997;11:55181.

23. Hooton TM. Recurrent urinary tract infection in women. Int J Antimicrob Agents 2001;17:259-68.

24. Foxman B. Recurring urinary tract infection: incidence and risk factors. Am J Public Health 1990;80:331-3.

25. Krieger JN. Urinary tract infections: what's new? J Urol 2002;168:23518.

26. Stamm WE, Hooton TM. Management of urinary tract infections in adults. N Engl J Med 1993;329:1328-34.

27. Svanborg C, Godaly G. Bacterial virulence in urinary tract infection. Infect Dis Clin North Am 1997;11:513-29.

28. Mulvey MA, Schilling JD, Martinez JJ, Hultgren SJ. Bad bugs and beleaguered bladders: interplay between uropathogenic Escherichia coli and innate host defenses. Proc Natl Acad Sci U S A 2000;97:8829-35.

29. Schilling JD, Mulvey MA, Hultgren SJ. Dynamic interactions between host and pathogen during acute urinary tract infections. Urology 2001;57(6 Suppl 1):56-61. 